\title{
Cancer antigen-125 and risk of atrial fibrillation: a systematic review and meta-analysis
}

\begin{abstract}
Angel Cheung, ${ }^{1}$ Mengqi Gong, ${ }^{2}$ Roberto Bellanti, ${ }^{3}$ Sadeq Ali-Hasan-Al-Saegh, ${ }^{4}$ Guangping Li, ${ }^{2}$ Eulàlia Roig, ${ }^{5}$ Julio Núñez, ${ }_{1}^{6,7}$ Thomas D Stamos, ${ }^{8}$ Mehmet Birhan Yilmaz, ${ }^{9}$ Kaya Hakki, ${ }^{9}$ William K K Wu, ${ }^{10,11}$ Sunny Hei Wong, ${ }^{11,12}$ Wing Tak Wong, ${ }^{13}$ George Bazoukis, ${ }_{14}$ Konstantinos Lampropoulos, ${ }_{1}^{14}$ Lah Ah Tse, ${ }^{15}$ Jichao Zhao ${ }_{16}^{16}$ Gregory Y H Lip, ${ }^{17,18}$ Adrian Baranchuk, ${ }^{19}$ Martin C S Wong, ${ }^{20}$ Tong Liu, ${ }^{2}$ Gary Tse, ${ }^{11,12}$ International Health Informatics Study (IHIS) Network
\end{abstract}

\begin{abstract}
- Additional material is
published online only. To view please visit the journal online (http://dx.doi.org/10.1136/ heartasia-2017-010970).
\end{abstract}

For numbered affiliations see end of article.

\section{Correspondence to} Dr Gary Tse, Department of Medicine and Therapeutics and Li Ka Shing Institute of Health Sciences, Faculty of Medicine, Chinese University of Hong Kong, Hong Kong, China; tseg@ cuhk.edu.hk

Received 16 October 2017 Revised 10 November 2017 Accepted 12 November 2017

\section{CrossMark}

To cite: Cheung A, Gong M, Bellanti $\mathrm{R}$, et al. Heart Asia 2018;10:1-6. doi:10.1136/ heartasia-2017-010970

\section{ABSTRACT \\ Background Cancer antigen-125 (Ca-125) is} traditionally recognised as a tumour marker and its role in cardiovascular diseases has been studied only in recent years. Whether Ca-125 is elevated in patients with atrial fibrillation $(\mathrm{AF})$ and its levels predict the risk of AF remains controversial. Therefore, we conducted a systematic review and meta-analysis of the association between Ca-125 levels and AF.

Methods PubMed and EMBASE databases were searched until 1 June 2017 for studies that evaluated the association between Ca-125 and AF. Inclusion criteria included studies that compare Ca-125 in patients with and without $A F$, or those reporting $H$ Rs/ORs for risk of $A F$ stratified by Ca-125 levels.

Results A total of 39 entries were retrieved from the databases, of which 10 studies were included in the final meta-analysis. Ca-125 was significantly higher in patients with AF compared with those in sinus rhythm (mean difference $=16 \mathrm{U} / \mathrm{mL}, 95 \% \mathrm{Cl} 2$ to $30 \mathrm{U} / \mathrm{mL}$, $\left.\mathrm{P}<0.05 ; I^{2}: 98 \%\right)$. Ca-125 significantly increased the risk of $A F$ (HR: $1.39,95 \% \mathrm{Cl} 1.06$ to $1.82, \mathrm{P}<0.05 ; \mathrm{I}^{2}$ : $84 \%$ ).

Conclusion Ca-125 was significantly higher in patients with AF than in those in sinus rhythm, and high Ca125 is predictive of AF occurrence. However, the high heterogeneity observed means there is an uncertainty in the relationship between $\mathrm{Ca}-125$ and $A F$, which needs to be confirmed by larger prospective studies.

\section{INTRODUCTION}

Atrial fibrillation (AF) is the most commonly observed arrhythmia encountered during clinical practice, but its pathogenesis is multifactorial, with recent work implicating inflammatory activation as an underlying mechanism. ${ }^{1}$ Several inflammatory markers, such as C-reactive protein and interleukin (IL)-6, are elevated in the context of $\mathrm{AF}^{2}{ }^{3}$ Recent work has focused on the roles of non-traditional markers in cardiovascular disorders. For example, cancer antigen-125 (Ca-125) is a tumour marker whose levels are typically raised in malignancies such as ovarian cancer. Ca-125 may be a secondary cytokine whose levels are increased in response to primary cytokines such as tumour necrosis factoralpha (TNF- $\alpha$ ) and IL- $1,{ }^{4}$ and thus is elevated in proinflammatory conditions. Since inflammation has been associated with heart failure (HF) and AF, $\mathrm{Ca}-125$ may be a useful biomarker for predicting disease severity and occurrence. Indeed, Ca-125 levels have been shown to correlate with symptoms and disease progression in $\mathrm{HF}^{5}$ This marker has been associated with higher risk of AF in some studies, while others have reported no significant association. Therefore, the aim of this systematic review and meta-analysis is to elucidate the relationship between Ca-125 levels and risk of AF.

\section{METHODS}

\section{Search strategy, inclusion and exclusion criteria}

The meta-analysis was performed according to the Preferred Reporting Items for Systematic Reviews and Meta-Analyses (PRISMA) statement. PubMed and EMBASE were searched for studies that investigated the relationship between $\mathrm{Ca}-125$ levels and AF using the following terms: (' $\mathrm{Ca}-125$ ' and 'atrial fibrillation'). The search period was from the beginning of the databases to 1 July 2017 , with no language restrictions. The following inclusion criteria were applied: (1) the design was a casecontrol, prospective or retrospective cohort study in humans; (2) Ca-125 values were provided and related to $\mathrm{AF}$ endpoints.

The quality assessment of these studies included in our meta-analysis was performed using the Newcastle-Ottawa Quality Assessment Scale (NOS). The point score system evaluated the categories of study participant selection, comparability of the results and quality of the outcomes. The following characteristics were assessed: (A) representativeness of the exposed cohort; (B) selection of the non-exposed cohort; (C) ascertainment of exposure; (D) demonstration that outcome of interest was not present at the start of study; (E) comparability of cohorts on the basis of the design or analysis; (F) assessment of outcomes; $(G)$ follow-up period sufficiently long for outcomes to occur; and $(\mathrm{H})$ adequacy of follow-up of cohorts. This scale varied from zero to nine stars, which indicated that studies were graded as poor quality if they met $<5$ criteria, fair if they met 5-7 criteria, and good if they met $>8$ criteria. The details of the NOS quality assessment are shown in online supplementary tables 1 and 2 .

\section{Data extraction and statistical analysis}

Data from the different studies were entered in prespecified spreadsheet in Microsoft Excel. All 
publications identified were assessed for compliance with the inclusion criteria. In this meta-analysis, the extracted data elements consisted of: (1) publication details: surname of first author, publication year; (2) study design; (3) follow-up duration; (4) the quality score; and (5) the characteristics of the population including sample size, gender, age and cut-off point for Ca-125 levels. This systematic review was conducted in accordance with PRISMA statement. ${ }^{6}$ Two reviewers (AC and GT) independently reviewed each included study and disagreements were resolved by adjudication with input from a third reviewer (TL).

Mean differences in Ca-125 levels between patients with and without AF were extracted from each study and subsequently pooled in our meta-analysis. For the relationship between Ca-125 and AF, multivariate adjusted HRs or ORs and their 95\% CIs were extracted from each study. When values from multivariate analysis were not available, those from univariate analysis were used. When the latter were not provided, raw data were used to calculate unadjusted HR where possible. ORs were converted to HRs using the formula $\mathrm{HR}=\mathrm{OR} /(1$ - baseline risk +(baseline risk $\times \mathrm{OR})$ ). Heterogeneity across studies was determined using Cochran's $Q$ value, which is the weighted sum of squared differences between individual study effects and the pooled effect across studies, and the $\mathrm{I}^{2}$ statistic from the standard $\chi^{2}$ test, which describes the percentage of the variability in the effect estimates resulting from heterogeneity. $\mathrm{I}^{2}>50 \%$ was considered to reflect significant statistical heterogeneity. The random effects model using the inverse variance heterogeneity method was used when $\mathrm{I}^{2}>50 \%$, otherwise the fixed effects model was used. To locate the origin of the heterogeneity, sensitivity analysis excluding one study at a time, and subgroup analyses based on different disease conditions and $\mathrm{Ca}-125$ cut-offs were performed. Funnel plots showing SEs or precision against the logarithms of the OR were constructed. Begg and Mazumdar rank correlation test and Egger's test were used to determine publication bias.

\section{RESULTS}

A flow diagram detailing the above search terms with inclusion and exclusion criteria is depicted in figure 1. A total of 9 and 30 entries were retrieved from PubMed and EMBASE, respectively. Comparing with the entries extracted from PubMed search, 12 duplicate entries from the EMBASE search were found and removed. This yielded 27 publications and further assessment demonstrated that 10 studies met the inclusion criteria. ${ }^{7-16}$

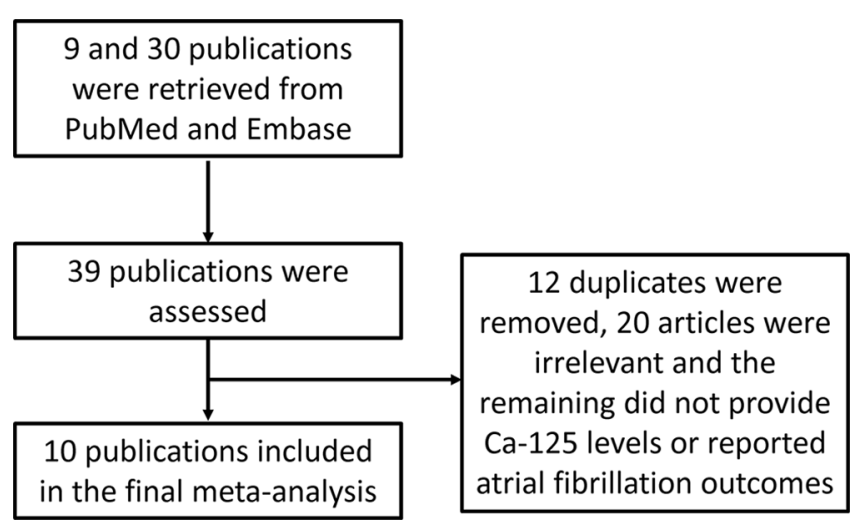

Figure 1 Flow chart of the database search and study selection process. Ca-125, cancer antigen-125.
Ca-125 levels for patients in AF and those in sinus rhythm were examined in the following contexts: HF in five studies, patients with cardiac symptoms in one study, medical inpatients who did not have HF, inflammatory or malignant conditions in three studies and postmenopausal women in one study. The baseline characteristics of these studies are listed in table 1. Five were prospective studies and five were retrospective studies. The mean follow-up duration was $32 \pm 34$ months. A total of 3858 subjects were included (mean: 386; range from 58 to 2642). The mean age was 71 years old ( $21 \%$ male). The mean cut-off point for $\mathrm{Ca}-125$ was $42.5 \pm 31.4 \mathrm{U} / \mathrm{mL}$.

\section{Ca-125 levels in AF versus sinus rhythm}

Nine studies compared Ca-125 levels between patients with AF and those in sinus rhythm. Of these, seven reported significant elevations in this marker with $\mathrm{AF}$, whereas two reported no difference when compared with controls (figure 2). The pooled mean difference was $16 \mathrm{U} / \mathrm{mL}(95 \% \mathrm{CI} 2$ to $20 \mathrm{U} / \mathrm{mL}, \mathrm{P}<0.05)$. $\mathrm{I}^{2}$ took a value of $98 \%$, indicating the presence of significant heterogeneity. Funnel plots are shown in online supplementary figures 1 and 2. Begg and Mazumdar rank correlation suggested no significant publication bias (Kendal's tau value $-0.13, \mathrm{P}>0.05$ ) Egger's test demonstrated no significant asymmetry (intercept $1.5, \mathrm{t}$-value $0.5 ; \mathrm{P}>0.05)$. Sensitivity analysis excluding one study at a time did not significantly affect the pooled estimate (online supplementary figure 3 ).

\section{Risk of AF from increased Ca-125 levels}

Eight studies examined the risk of incident $\mathrm{AF}$ as a function of Ca-125 levels. Of these, five studies reported a higher risk of AF with elevations in $\mathrm{Ca}-125$, whereas three studies reported no significant increase in this risk. The pooled meta-analysis demonstrated that elevated $\mathrm{Ca}-125$ levels are associated with 1.39 times higher risk of AF (95\% CI 1.06 to $1.82, \mathrm{P}<0.05$; figure 3 ). $\mathrm{I}^{2}$ took a value of $84 \%$, indicating the presence of substantial heterogeneity. Funnel plots are shown in online supplementary figures 4 and 5. Begg and Mazumdar rank correlation suggested no significant publication bias (Kendal's tau value $0.18, \mathrm{P}>0.05$ ). Egger's test demonstrated significant asymmetry (intercept 1.9, t-value $3.0 ; \mathrm{P}<0.01)$. Sensitivity analysis excluding one study at a time did not significantly affect the pooled estimate (online supplementary figure 6). Next, subgroup analysis based on different Ca- 125 cut-off values was performed to determine whether this was a source of heterogeneity. However, meta-analysis of studies using $\leq 35 \mathrm{U} / \mathrm{mL}$ demonstrated a pooled HR of $1.26(95 \% \mathrm{CI}$ 0.96 to $\left.1.66 ; \mathrm{P}=0.1 ; \mathrm{I}^{2}=0 \%\right)$ and $>35 \mathrm{U} / \mathrm{mL}$ demonstrated a pooled HR of 1.49 (95\% CI 1.06 to $2.08 ; \mathrm{P}<0.05 ; \mathrm{I}^{2}=88 \%$ ). Given the high heterogeneity that remains on pooling the studies using a cut-off of $>35 \mathrm{U} / \mathrm{mL}$, different cut-off values did not appear to contribute significantly to the heterogeneity observed.

\section{Subgroup analyses based on underlying medical conditions}

To locate the origin of the heterogeneity, subgroup analyses based on the different disease conditions were performed. First, five studies on HF were included. The pooled mean difference for $\mathrm{Ca}-125$ between patients with $\mathrm{HF}$ with $\mathrm{AF}$ and patients with $\mathrm{HF}$ in sinus rhythm was $29 \mathrm{U} / \mathrm{mL}(95 \% \mathrm{CI} 1$ to $56 \mathrm{U} / \mathrm{mL} ; \mathrm{I}^{2}: 87 \%$ ) (online supplementary figure 7 ). The HR for $\mathrm{AF}$ risk was 1.20 but this did not quite reach significance (95\% CI 0.93 to $1.54, \mathrm{P}>0.05 ; \mathrm{I}^{2}: 75 \%$ ) (online supplementary figure 8 ). Second, three studies on general medical inpatients excluding HF, inflammatory conditions and malignancy were meta-analysed. This demonstrated no significant difference in $\mathrm{Ca}-125$ values between patients with $\mathrm{AF}$ and those in sinus 


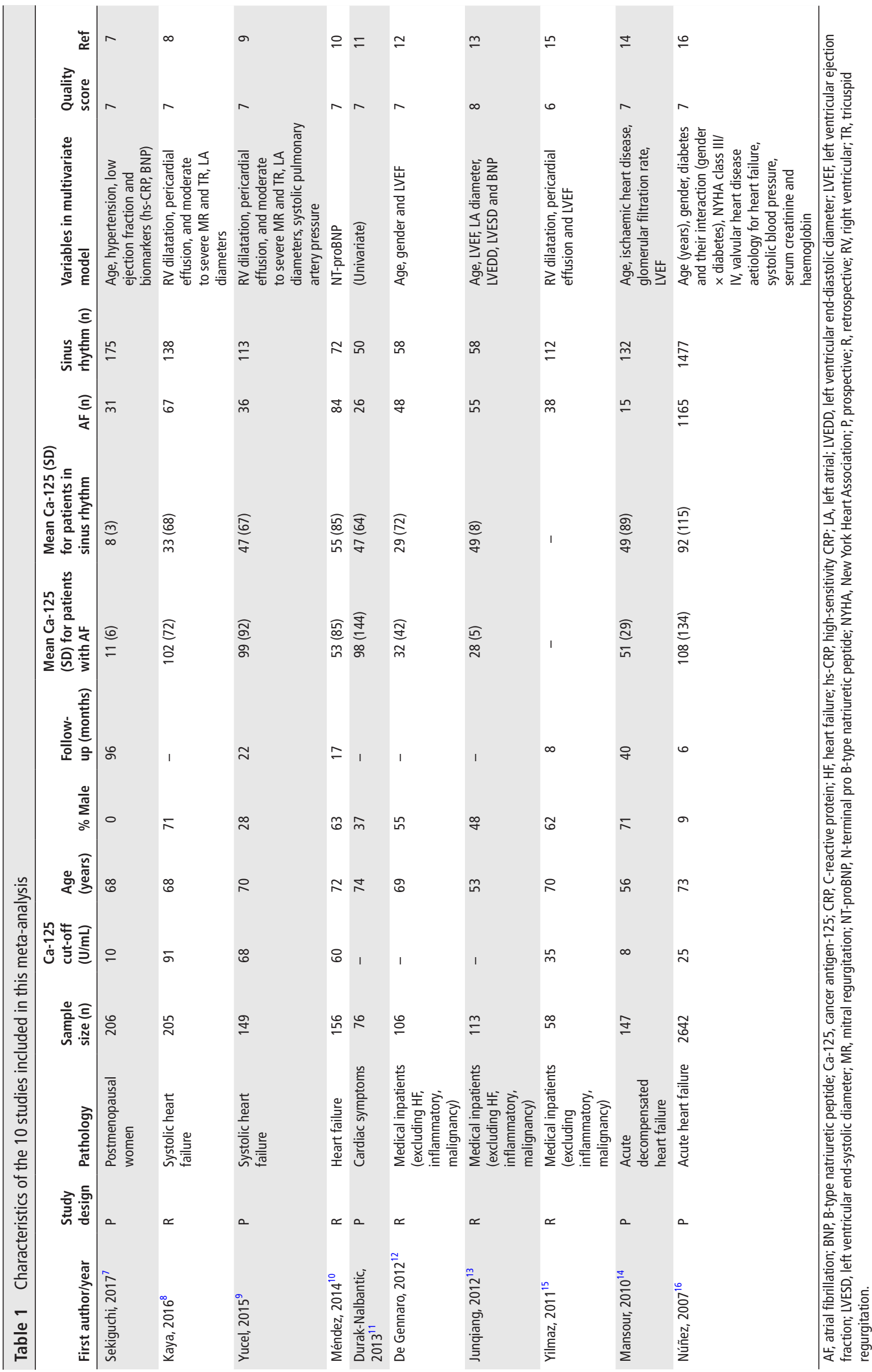




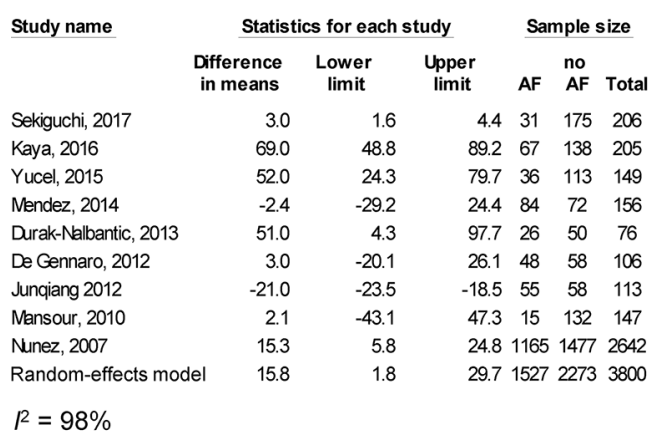

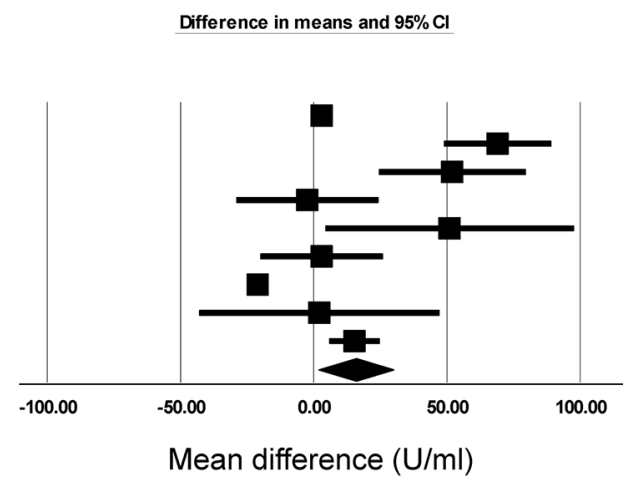

Lower in AF Higher in AF

Figure 2 Mean difference in cancer antigen-125 (Ca-125) levels between patients with atrial fibrillation and those in sinus rhythm. AF, atrial fibrillation.

rhythm (mean difference $=-12 \mathrm{U} / \mathrm{mL}, 95 \% \mathrm{CI}-35$ to $11 \mathrm{U} / \mathrm{mL}$; $\mathrm{I}^{2}: 76 \%$ ) (online supplementary figure 9). The HR for AF risk was 2.25 but this did not reach significance $(95 \% \mathrm{CI} 0.84$ to $\left.6.04, \mathrm{P}>0.05 ; \mathrm{I}^{2}: 89 \%\right)$ (online supplementary figure 10 ).

\section{DISCUSSION}

The main findings of this meta-analysis are that: (1) Ca-125 levels in patients with $\mathrm{AF}$ were higher than those who were with sinus rhythm; and (2) increased Ca-125 levels are associated with an increased risk of AF.

Ca-125 (also called carbohydrate antigen) is produced by coelomic epithelial cells, such as those located in the peritoneum and pleura, after mechanical stress and/or inflammation. ${ }^{17}$ Classically, Ca-125 was studied as a tumour marker associated with ovarian cancer. Increases in serum levels of $\mathrm{Ca}-125$ have been described in women with ovarian cancer associated with peritoneal involvement as well as, although less frequently, in other types of cancer (lung, breast, uterine and gastrointestinal tract). Ca-125 can be elevated also in nephrotic syndrome, hepatic cirrhosis and HF. Ca-125 is therefore a non-specific marker. Recently, it has been implicated in cardiovascular disease, with increased plasma Ca-125 concentration having been reported in patients suffering from pericardial, pleural and peritoneal effusions. ${ }^{12} \mathrm{~A}$ recent meta-analysis investigated the predictive value of $\mathrm{Ca}-125$ in $\mathrm{HF},{ }^{18}$ but its value in $\mathrm{AF}$ remains controversial. For example, some studies reported higher values of Ca-125 in subjects with AF compared with those in sinus rhythm, whereas others reported no significant difference. Previous studies have associated AF with increased cytokine levels ${ }^{19}$; further consideration of plasma $\mathrm{Ca}-125$ levels may thus serve as an important measure of risk of developing $\mathrm{AF}$ as well as a useful marker in $\mathrm{AF}$ burden-reducing strategies. Higher levels of Ca-125 in patients with $\mathrm{HF}$ with $\mathrm{AF}$ were speculated to be linked to increased stress and inflammation. ${ }^{20}$ The mechanisms underlying the pathogenesis of AF, such as inflammation, increased oxidative stress and a prothrombotic state, may be decreased through timely diagnosis and quicker application of appropriate treatment. This might prove $\mathrm{Ca}-125$ levels to be a valuable prognostic tool, allowing for targeted treatment approaches, with it already being a strong predictor of mortality in patients with decompensated $\mathrm{HF}^{21}$

The mechanisms underlying raised $\mathrm{Ca}-125$ levels in cardiovascular diseases, including AF, are manyfold. Impaired ventricular function, left atrial remodelling, increased vascular mechanical and oxidative stress, ${ }^{22}$ a prothrombotic state and overall systemic inflammatory response lead to a significant increase in the production of cytokines such as IL- 6 , TNF- $\alpha$, IL-8 and IL-10,

High Ca-125 levels and AF

\begin{tabular}{lclll} 
Study name & \multicolumn{4}{c}{ Statistics for each study } \\
& $\begin{array}{c}\text { Hazard } \\
\text { ratio }\end{array}$ & $\begin{array}{c}\text { Lower } \\
\text { limit }\end{array}$ & $\begin{array}{c}\text { Upper } \\
\text { limit }\end{array}$ & p-Value \\
Sekiguchi, 2017 & 1.287 & 0.972 & 1.703 & 0.078 \\
Kaya, 2016 & 1.007 & 1.002 & 1.013 & 0.012 \\
Yucel, 2015 & 2.693 & 1.285 & 5.642 & 0.009 \\
Mendez, 2014 & 1.005 & 0.715 & 1.413 & 0.977 \\
Yilmaz, 2011 & 3.870 & 2.105 & 7.115 & 0.000 \\
Mansour, 2010 & 0.753 & 0.179 & 3.160 & 0.698 \\
Nunez, 2007 & 1.382 & 1.125 & 1.697 & 0.002 \\
Random-effects model & 1.392 & 1.063 & 1.822 & 0.016 \\
$\quad$ P $=84 \%$ & & & &
\end{tabular}

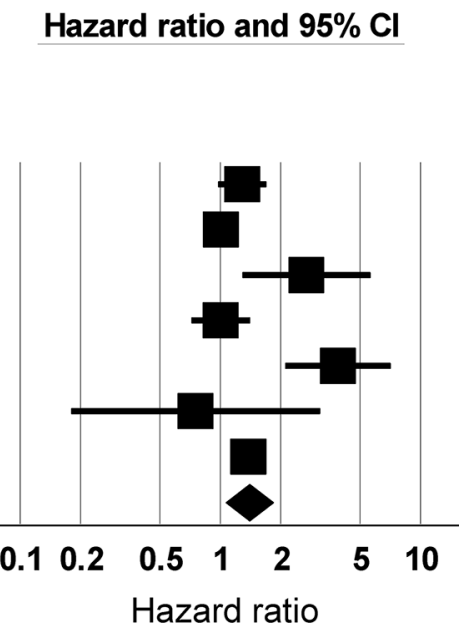

Lower risk Higher risk

Figure 3 HR for atrial fibrillation risk due to increased Ca-125 levels. AF, atrial fibrillation; Ca-125, cancer antigen-125. 
which induce release of Ca-125 from the epithelial coelomic cells of the serosal tissues. Although most of the studies included in this meta-analysis showed significantly increased levels of Ca-125 in patients with AF, the actual relationship between $\mathrm{Ca}-125$ and AF remains not entirely clear. Increases in this non-specific marker could at the same time reflect a surrogate for the progression of underlying diseases, play an active role in worsening cardiac function in patients with HF and significantly contribute to AF development.

In addition, in patients with permanent $\mathrm{AF}$, higher heart rates and larger left atrium dimensions were also correlated with high Ca-125 levels with concomitant increases in neuroendocrine messengers such as norepinephrine and atrial natriuretic peptides. ${ }^{23}$ These findings, in combination with results from this meta-analysis, may shed some light into the pathophysiological mechanism stimulating $\mathrm{Ca}-125$ production within patients with AF.

\section{Strengths and limitations}

The main strengths of this meta-analysis are as follows. First, PRISMA guidelines were adhered to, and therefore we are confident that the quality of this study is high. Second, 9 of the 10 studies had a score of 7 or above out of 10 for quality analysis, indicating that the data from these studies were reliable. However, some limitations remain. First, Ca-125 elevation might be associated with undiagnosed underlying comorbidity, the prevalence of which might have been underestimated in this meta-analysis. Our subgroup analyses demonstrated that $\mathrm{Ca}-125$ levels remained higher in patients with AF compared with those in sinus rhythm in HF, but not in medical inpatients without HF, inflammatory conditions and malignancy. This would suggest $\mathrm{HF}$ as an underlying cause of $\mathrm{Ca}-125$ release. Although in each study patients with previous diagnosis of malignancy were excluded, there could have been cases of occult or undiagnosed malignancy, which remains a potential confounder. Nevertheless, the included studies had high quality scores, increasing the confidence of the data used for our meta-analysis.

Second, attempts were made to identify the origin of the high heterogeneity for our meta-analysis of HRs for mortality. The nature of the HRs (ie, multivariate or univariate values) did not contribute significantly to heterogeneity since all studies apart from one used multivariate HRs. High heterogeneity remained on pooling the studies using different Ca-125 cut-off values ( $\leq 35$ vs $>35 \mathrm{U} / \mathrm{mL}$ ). However, $\mathrm{I}^{2}$ remained substantial for cut-off $>35 \mathrm{U} / \mathrm{mL}$, taking a value of $88 \%$, suggesting that the differential cut-offs did not contribute significantly to the heterogeneity observed. Subgroup analysis for HF yielded also $\mathrm{I}^{2}$ of $75 \%$, indicating that even within the HF cohorts, the populations were heterogeneous. Moreover, to explore for the high heterogeneity for mean difference of $\mathrm{Ca}-125$ between patients with $\mathrm{AF}$ and non-AF patients, subgroup analyses based on $\mathrm{HF}$ and general medical conditions were conducted. However, $\mathrm{I}^{2}$ remained substantial in both cases, taking values of $87 \%$ and $76 \%$, respectively, suggesting the different patient populations did not contribute significantly to the heterogeneity observed.

Third, sample size is a limitation. Some studies included small numbers of patients and some subgroups were not adequately represented (ie, AF lasting $<6$ months), thus potentially biasing the outcomes. Results from these studies could have been confirmed in larger cohorts of patients and in prospective studies. Importantly, in some studies, a single ECG was used to detect AF, rather than a prolonged monitoring method, such as 48 -hour Holter ECG. This may have significantly underestimated the incidence of asymptomatic $\mathrm{AF}$.
Finally, different commercially available kits with different reference ranges were used to determine Ca-125 levels. The majority used a solution of suspended, submicron-sized latex particles to measure analytes. However, clinical performance for measurement of $\mathrm{Ca}-125$ elevation has not been compared and this represents a potential limitation. Further efforts are needed to standardise Ca-125 measurement and reduce assay variability.

\section{CONCLUSIONS}

Our meta-analysis found that Ca-125 was associated with AF, but due to the high heterogeneity between the studies, larger prospective studies are needed to confirm the relationship and its value as a biomarker.

\section{Key messages}

What is already known about this subject? Cancer antigen-125 (Ca-125) is associated with increased cardiovascular risk. It is also associated with atrial fibrillation (AF) in some studies but not others.

\section{What does this study add?}

Ca-125 is higher in patients with AF than those in sinus rhythm, and may be a predictor of AF. Ca-125 is especially high in patients with $\mathrm{AF}$ with heart failure.

\section{How might this impact on clinical practice?}

Ca-125 may be a novel biomarker of AF. However, due to the high heterogeneity between the studies, larger prospective studies are needed to confirm the relationship and its value as a biomarker.

\section{Author affiliations}

'Department of Biomedical Engineering, Brown University, Providence, Rhode Island, USA

${ }^{2}$ Department of Cardiology, Tianjin Key Laboratory of Ionic-Molecular Function of Cardiovascular Disease, Tianjin Institute of Cardiology, Second Hospital of Tianjin Medical University, Tianjin, China

${ }^{3}$ Department of Medicine, Cambridge University Hospital NHS Trust, Cambridge, UK ${ }^{4}$ Cardiovascular Research Center, Shahid Sadoughi University of Medical Sciences, Yazd, Iran

${ }^{5}$ Cardiology Department, Hospital de la Santa Creu i Sant Pau, Institut de Recerca

Biomèdica Sant Pau and Universitat Autònoma, Barcelona, Spain

${ }^{6}$ Cardiology Department, Departamento de Medicina, Hospital Clínico Universitario, Universitat de València, Valencia, Spain

${ }^{7}$ CIBER in Cardiovascular Diseases (CIBERCV), Madrid, Spain

${ }^{8}$ Department of Cardiology, University of Illinois at Chicago, Chicago, Illinois, USA

${ }^{9}$ Department of Cardiology, Cumhuriyet University Medical School, Sivas, Turkey

${ }^{10}$ Department of Anaesthesia and Intensive Care, Faculty of Medicine, Chinese

University of Hong Kong, Hong Kong, China

${ }^{11}$ Faculty of Medicine, Li Ka Shing Institute of Health Sciences, Chinese University of Hong Kong, Hong Kong, China

${ }^{12}$ Department of Medicine and Therapeutics, Faculty of Medicine, Chinese University

of Hong Kong, Hong Kong, China

${ }^{13}$ School of Life Sciences, Chinese University of Hong Kong, Hong Kong, China

${ }^{14}$ Second Department of Cardiology, Laboratory of Cardiac Electrophysiology,

Evangelismos General Hospital of Athens, Athens, Greece

${ }^{15}$ Division of Occupational and Environmental Health, JC School of Public Health and

Primary Care, The Chinese University of Hong Kong, Hong Kong, China

${ }^{16}$ Auckland Bioengineering Institute, The University of Auckland, Auckland, New Zealand

${ }^{17}$ Institute of Cardiovascular Sciences, University of Birmingham, Birmingham, UK

${ }^{18}$ Aalborg Thrombosis Research Unit, Department of Clinical Medicine, Aalborg University, Aalborg, Denmark

${ }^{19}$ Division of Cardiology, Kingston General Hospital, Queen's University, Kingston, Ontario, Canada

${ }^{20} \mathrm{JC}$ School of Public Health and Primary Care, The Chinese University of Hong Kong, Hong Kong, China 
Contributors AC: screening of articles, data extraction, drafting of manuscript. MG: critical revision of manuscript, conducted quality analysis. GT: conceived the study, screening of articles, data extraction, drafted the manuscript, critical revision of manuscript. JN, TDS, MBY, KH: provided additional data from their original publications for meta-analysis, critical revision of manuscript. All other authors contributed to critical revision of manuscript.

Funding GT and SHW are supported by the Croucher Foundation of Hong Kong. This work was supported by a research grant (Number: 81570298) from the National Natural Science Foundation of China to TL.

Competing interests None declared.

Provenance and peer review Not commissioned; externally peer reviewed.

(c) Article author(s) (or their employer(s) unless otherwise stated in the text of the article) 2018. All rights reserved. No commercial use is permitted unless otherwise expressly granted.

\section{REFERENCES}

1 Hu YF, Chen YJ, Lin YJ, et al. Inflammation and the pathogenesis of atrial fibrillation. Nat Rev Cardiol 2015;12:230-43.

2 Chung MK, Martin DO, Sprecher D, et al. C-reactive protein elevation in patients with atrial arrhythmias: inflammatory mechanisms and persistence of atrial fibrillation. Circulation 2001;104:2886-91.

3 Conway DS, Buggins P, Hughes E, et al. Relationship of interleukin-6 and C-reactive protein to the prothrombotic state in chronic atrial fibrillation. J Am Coll Cardiol 2004;43:2075-82

4 Kosar F, Aksoy Y, Ozguntekin G, et al. Relationship between cytokines and tumour markers in patients with chronic heart failure. Eur J Heart Fail 2006;8:270-4.

5 Kouris NT, Zacharos ID, Kontogianni DD, et al. The significance of CA125 levels in patients with chronic congestive heart failure. Correlation with clinical and echocardiographic parameters. Eur J Heart Fail 2005;7:199-203.

6 Moher D, Liberati A, Tetzlaff J, et al. Preferred reporting items for systematic reviews and meta-analyses: the PRISMA statement. Ann Intern Med 2009;151:264-9.

7 Sekiguchi H, Shimamoto K, Takano M, et al. Cancer antigen-125 plasma level as a biomarker of new-onset atrial fibrillation in postmenopausal women. Heart 2017; 103:1368-73.

8 Kaya $\mathrm{H}$, Zorlu A, Yucel $\mathrm{H}$, et al. Higher cancer antigen 125 level is associated with the presence of permanent atrial fibrillation in systolic heart failure patients. Acta Cardiol 2016;71:61-6.
9 Yucel $\mathrm{H}$, Kaya $\mathrm{H}$, Zorlu A, et al. Cancer antigen 125 levels and increased risk of newonset atrial fibrillation. Herz 2015;40(Suppl 2):119-24.

10 Méndez $A B$, Ordoñez-Llanos J, Ferrero A, et al. Prognostic value of increased carbohydrate antigen in patients with heart failure. World I Cardiol 2014;6:205-12.

11 Durak-Nalbantic A, Resic N, Kulic M, et al. Serum level of tumor marker carbohydrate antigen-CA125 in heart failure. Med Arch 2013;67:241-4.

12 De Gennaro L, Brunetti ND, Montrone D, et al. Inflammatory activation and carbohydrate antigen-125 levels in subjects with atrial fibrillation. Eur J Clin Invest 2012;42:371-5

13 Junqiang $P$, Dianxin Z, Zaiwei Z, et al. Serum carbohydrate antigen-125 is elevated in patients with chronic atrial fibrillation. Journal of Medical Colleges of PLA 2012;27:286-93.

14 Mansour IN, Napan S, Tarek Alahdab M, et al. Carbohydrate antigen 125 predicts long-term mortality in African American patients with acute decompensated heart failure. Congest Heart Fail 2010;16:15-20.

15 Yilmaz MB, Zorlu A, Tandogan I. Plasma CA-125 level is related to both sides of the heart: a retrospective analysis. Int I Cardiol 2011;149:80-2.

16 Núñez J, Núñez E, Consuegra L, et al. Carbohydrate antigen 125: an emerging prognostic risk factor in acute heart failure? Heart 2007;93:716-21.

17 Barbieri RL, Niloff JM, Bast RC, et al. Elevated serum concentrations of CA-125 in patients with advanced endometriosis. Fertil Steril 1986;45:630-4.

18 Zhuang J, Faggiano P, Li Q, et al. Insights into the clinical implications of carbohydrate antigen 125 as a biomarker of heart failure: a meta-analysis and systematic review of published studies. J Cardiovasc Med 2014;15:864-72.

19 O'Brien TJ, Tanimoto H, Konishi I, et al. More than 15 years of CA 125: what is known about the antigen, its structure and its function. Int I Biol Markers 1998;13:188-95.

20 Aviles RJ, Martin DO, Apperson-Hansen C, et al. Inflammation as a risk factor for atria fibrillation. Circulation 2003;108:3006-10.

21 D'Aloia A, Faggiano P, Aurigemma G, et al. Serum levels of carbohydrate antigen 125 in patients with chronic heart failure: relation to clinical severity, hemodynamic and Doppler echocardiographic abnormalities, and short-term prognosis. J Am Coll Cardiol 2003:41:1805-11.

22 Tse G, Yan BP, Chan YW, et al. Reactive Oxygen Species, Endoplasmic Reticulum Stress and Mitochondrial Dysfunction: The Link with Cardiac Arrhythmogenesis. Front Physiol 2016;7:313.

23 Hülsmann M, Berger R, Mörtl D, et al. Incidence of normal values of natriuretic peptides in patients with chronic heart failure and impact on survival: a direct comparison of $\mathrm{N}$-terminal atrial natriuretic peptide, $\mathrm{N}$-terminal brain natriuretic peptide and brain natriuretic peptide. Eur J Heart Fail 2005;7:552-6. 